\title{
The cadherin-catenin adhesion system in signaling and cancer
}

\author{
Maralice Conacci-Sorrell, Jacob Zhurinsky, and Avri Ben-Ze'ev \\ Department of Molecular Cell Biology, The Weizmann Institute of Science, Rehovot, Israel \\ Address correspondence to: Avri Ben-Ze'ev, Department of Molecular Cell Biology, \\ The Weizmann Institute of Science, Rehovot 76100, Israel. \\ Phone: 972-8-9342422; Fax: 972-8-9465261; E-mail: avri.ben-zeev@weizmann.ac.il. \\ Maralice Conacci-Sorrell and Jacob Zhurinsky contributed equally to this work. \\ J. Clin. Invest. 109:987-991 (2002). DOI:10.1172/JCI200215429.
}

The adhesion of cells to their neighbors determines cellular and tissue morphogenesis and regulates major cellular processes including motility, growth, differentiation, and survival. Cell-cell adherens junctions (AJs), the most common (indeed, essentially ubiquitous) type of intercellular adhesions, are important for maintaining tissue architecture and cell polarity and can limit cell movement and proliferation. AJs assemble via homophilic interactions between the extracellular domains of calcium-dependent cadherin receptors on the surface of neighboring cells. The cytoplasmic domains of cadherins bind to the submembranal plaque proteins $\beta$-catenin or plakoglobin ( $\gamma$-catenin), which are linked to the actin cytoskeleton via $\alpha$-catenin (Figure 1 ; refs. 1 , 2 ). The transmembrane assembly of cadherin receptors with the cytoskeleton is necessary for the stabilization of cell-cell adhesions and normal cell physiology.

Malignant transformation is often characterized by major changes in the organization of the cytoskeleton, decreased adhesion, and aberrant adhesion-mediated signaling. Disruption of normal cell-cell adhesion in transformed cells may contribute to tumor cells' enhanced migration and proliferation, leading to invasion and metastasis. This disruption can be achieved by downregulating the expression of cadherin or catenin family members or by activation of signaling pathways that prevent the assembly of AJs. The importance of the major epithelial cell cadherin, E-cadherin (E-cad, the product of the $\mathrm{CDH} 1$ gene), in the maintenance of normal cell architecture and behavior is underscored by the observation that hereditary predisposition to gastric cancer results from germline mutations in $\mathrm{CDH}$. Loss of E-cad expression eliminates $\mathrm{AJ}$ formation and is associated with the transition from adenoma to carcinoma and acquisition of metastatic capacity (3). Reestablishment of AJs in cancer cells by restoration of cadherin expression (4) exerts tumor-suppressive effects, including decreased proliferation and motility. In this Perspective, we discuss the molecular mechanisms underlying the role of the cadherin-catenin system in the regulation of cell proliferation, invasion, and intracellular signaling during cancer progression.

\section{Downregulation of $\mathrm{A}$ J assembly by mutations, hypermethylation, and transcriptional repression of E-cad expression}

Mutations in CDH1 that compromise the adhesive function of E-cad have been observed in human gastric carcinoma cell lines, lobular breast cancer, and familial gastric cancer (5). Certain tumors, for example invasive lobular carcinoma of the breast, and tumor cell lines that display mutations in one allele of $\mathrm{CDH} 1$ also acquire a deletion in the other allele, consistent with a two-hit mechanism for the loss of E-cad and suggesting that $C D H 1$ behaves as a classical tumor suppressor gene. While acquisition of loss-of-function mutations and the subsequent loss of heterozygosity are important mechanisms for silencing E-cad expression in tumor cells, progression to the metastatic phenotype can also involve a reversible downregulation of E-cad expression at the transcriptional level, sometimes achieved by methylation of the $\mathrm{CDH} 1$ promoter.

DNA methylation often causes downregulation of tumor suppressor genes in cancer cells by changing chromatin structure, thereby making the DNA inaccessible for transcription factors and RNA polymerase II (6). Hypermethylation of the $C D H 1$ promoter has been observed in human breast, prostate, and hepatocellular tumors that carry a wild-type $C D H 1$ gene. This methylation is reversible and can vary according to changes in the tumor microenvironment. For example, Graff et al. (7) found that when primary cultures of human breast carcinoma cells displaying a methylated $C D H 1$ promoter were cultured as spheroids, which requires homotypic cell-cell adhesion, promoter methylation decreased, allowing expression of E-cad. In some cases, for example in patients with hereditary diffuse gastric cancers that carry germline mutations in one allele of the $\mathrm{CDH} 1$ gene, the remaining allele is inactivated by DNA methylation (8). Finally, inhibition of DNA methylation can suppress the initiation of tumor development in a mouse model system for colorectal cancer, suggesting that methylation provides an attractive target for anticancer therapy.

Transcriptional silencing of $C D H 1$ may also result from aberrant expression of transcription factors that 


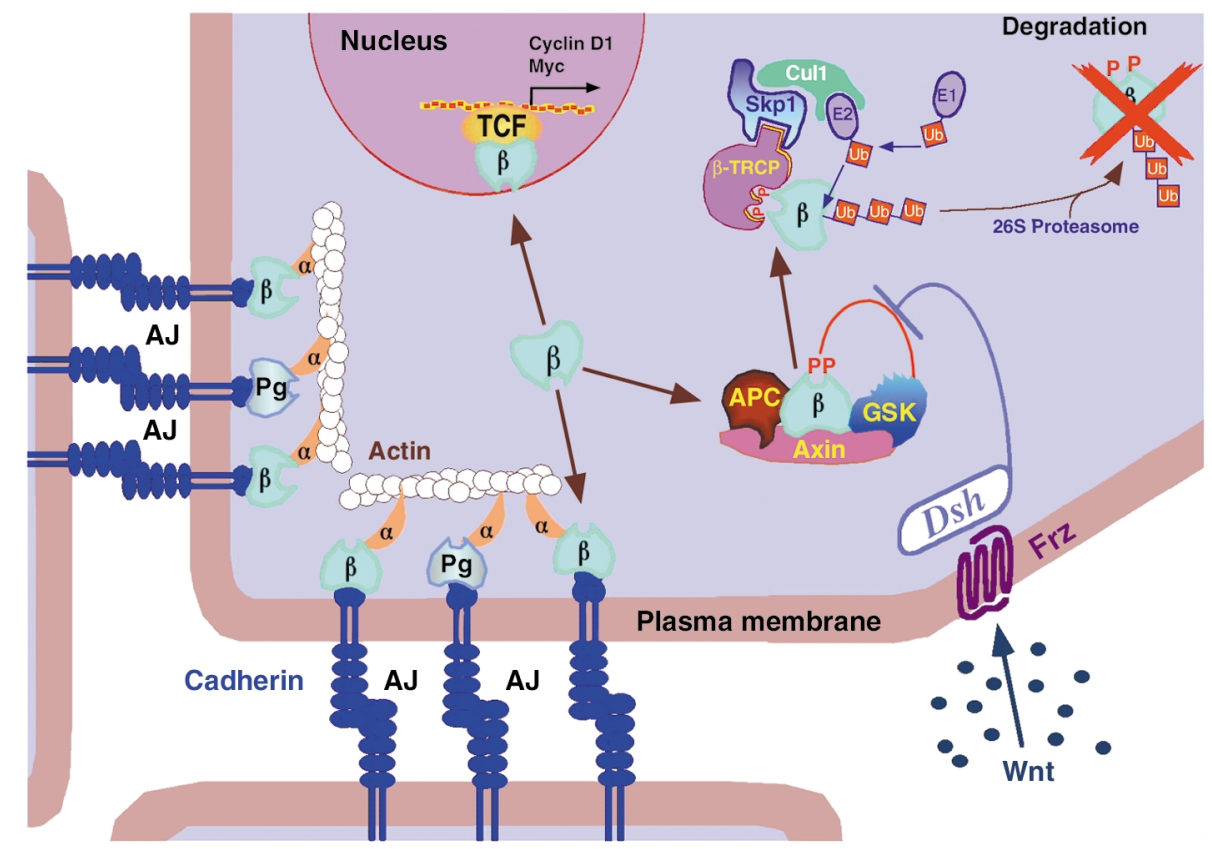

\section{Figure 1}

The dual role of $\beta$-catenin in cell adhesion and transcriptional activation. $\beta$-Catenin $(\beta)$ and plakoglobin $(\mathrm{Pg})$ bind to cadherin adhesion receptors, and via $\alpha$-catenin $(\alpha)$ they associate with the actin cytoskeleton to form AJs. When the Wnt signaling pathway is inactive, free $\beta$-catenin is degraded by a complex including glycogen synthase kinase (GSK), adenomatous polyposis coli (APC), and Axin, which phosphorylate $\beta$-catenin (PP). This protein complex recruits $\beta-\operatorname{TrCP}$, which, together with Skp1, Cul1, and the E1 and E2 ubiquitination components, mediates the ubiquitination of $\beta$-catenin $(\mathrm{Ub})$ and directs it to degradation by the $26 \mathrm{~S}$ proteasome. The binding of Wnt to Frizzled (Frz) receptors activates Wnt signaling, and disheveled (Dsh) inhibits $\beta$-catenin phosphorylation by GSK. This results in $\beta$-catenin accumulation in the nucleus, where it complexes with T cell factor (TCF) and transactivates target genes such as Cyclin D1 and Myc. Modified from ref. 56.

repress its promoter. These include snail, E12/E47, and SIP1, which bind to the $C D H 1$ promoter, inhibit E-cad transcription, and induce an epithelial-to-mesenchymal transition (EMT), leading to acquisition of invasiveness (9-12). The transcriptional repressor snail, a zinc finger protein originally identified as a regulator of mesoderm formation in developing Drosophila, was recently shown to be also critical for EMT in mouse development. In human carcinoma and melanoma, the expression of snail correlates with the absence of E-cad expression. Forced expression of snail in cultured epithelial cells represses the CDH1 promoter and induces a program of invasive growth characterized by the loss of AJs and the induction of EMT and tumorigenesis $(9,10)$. Interestingly, the invasive areas of mouse skin tumors express the highest levels of snail, supporting the view that activation of snail confers invasive properties on cells (9). E12/E47 and SIP1, which also repress the $C D H 1$ promoter, can likewise induce EMT, increase cell motility and invasion, and confer tumorigenicity. Moreover, SIP1 expression is elevated in several E-cad-negative human carcinoma cells (11). Thus, cancer cells employ reversible or irreversible mechanisms to silence the expression of E-cad.

These mechanisms may contribute to enhanced cancer cell motility and invasiveness. The transcriptional mechanisms involved in downregulation of E-cad are similar to those characteristic of typical stages during embryonic development, when changes in cell adhesion and motility take place. Since the loss of E-cad expres- sion is a common feature in many types of carcinoma, and since E-cad reintroduction into cancer cells reduces invasion and metastasis, cancer therapies targeting the regulation of E-cad expression may offer powerful means to control the spread of cancer. A recent study demonstrated that E-cad mRNA expression and cellcell adhesion are induced in SW480 human colon cancer cells by vitamin D3 (13). This makes vitamin D3 a potential candidate for treating colon cancer by inhibiting cell growth, similar to its effect on melanoma and soft tissue sarcoma.

\section{Regulation of catenins and AJ assembly in cancer cells}

The interaction between the cytoplasmic tail of cadherins with the catenins and the actin cytoskeleton (Figure 1; ref. 2) is critical for the establishment of stable and functional AJs. Catenin mutations that prevent this interaction have been reported in different types of cancer. A homozygous deletion of the $\alpha$-catenin gene, CTNNA1, leading to loss of cell-cell adhesion, has been found in a human lung cancer cell line (14). Introduction of wild-type $\alpha$-catenin into these cells restores normal adhesion. Likewise, AJ formation is blocked in an ovarian carcinoma cell line with a deletion in $\alpha$-catenin that abolishes binding to $\beta$-catenin (15). Expression of full-length $\alpha$-catenin in these cells restores their epithelial morphology, reduces their growth rate, and decreases their tumorigenic capacity (15). Deletions in the $\alpha$-catenin binding site of $\beta$-catenin have been iden- 
tified in a signet ring cell carcinoma of the stomach. In these cells, $\beta$-catenin-cadherin complexes are formed, but the lack of $\alpha$-catenin in these complexes prevents their linkage to the actin cytoskeleton and the assembly of AJs. Moreover, in mice where CTNNA1 is eliminated in the epidermis, AJs are disrupted and the epithelial skin cells display hyperproliferation (16). This hyperproliferation has been attributed to aberrant adhesion-mediated signaling, indicating that disruption of AJ assembly directly contributes to oncogenesis.

\section{The reciprocal relationship between cadherin- mediated adhesion and $\beta$-catenin signaling}

In addition to providing a physical link between cells, the mode of AJ assembly can influence various signaling pathways. A major route for signal transduction by AJs involves the regulation of $\beta$-catenin-T cell factor (TCF) signaling. $\beta$-Catenin plays a dual role in the cells: In addition to its structural role in $\mathrm{AJs}, \beta$-catenin can act as a transcription factor in the nucleus by serving as a coactivator of the lymphoid enhancer factor (LEF)/TCF family of DNA-binding proteins (Figure 1; ref. 17). $\beta$-Catenin-mediated transcription is activated by the Wnt pathway, which is crucial during various stages of embryonal development. Activation of Wnt signaling involves the inhibition of $\beta$-catenin degradation by the proteasomes, resulting in its nuclear accumulation and transcriptional activation of LEF/TCF target genes (Figure 1). Mutations in components that regulate $\beta$-catenin turnover (e.g., adenomatous polyposis coli [APC] or Axin) (Figure 1), as well as N-terminal mutations in $\beta$-catenin itself that compromise the protein's degradation, have been found in a variety of human cancers (18). These mutations cause $\beta$-catenin to accumulate in the nucleus and activate target genes in an aberrant manner. Since the interaction of $\beta$-catenin with cadherins and LEF/TCF family members is mediated by the same domain (the so-called arm repeat) on the $\beta$-catenin molecule, these interactions are mutually exclusive. Thus, recruitment of $\beta$-catenin into AJs by elevating the expression of cadherin can decrease its nuclear pool and antagonize $\beta$-cateninLEF/TCF transactivation (19-22).

$\beta$-Catenin can activate genes that stimulate cell proliferation, such as Cyclin D1 $(23,24)$ and Myc $(25)$, whose promoters contain LEF/TCF-binding sites. Downregulation of $\beta$-catenin-LEF/TCF signaling may therefore lead to decreased proliferation, as demonstrated in experiments where cadherin-dependent inhibition of growth was suggested to involve the sequestration of the signaling pool of $\beta$-catenin $(26,27)$. Another means to antagonize aberrant $\beta$-catenin signaling would be to enhance the antiproliferative p53-mediated response. Indeed, recent studies demonstrate that $\mathrm{p} 53$ expression is induced in cells displaying abnormal $\beta$-catenin levels (28). This induction is mediated by the elevation of p19ARF and is analogous to the cellular response to other oncoproteins, such as Ras and Myc (29). In addition to growth arrest, cells with aberrant $\beta$-catenin signaling show enhanced turnover of $\beta$-catenin, again as a result of elevated p53 expression (30-32).
Disruption of AJs releases $\beta$-catenin from the AJ pool. When it is not rapidly degraded, $\beta$-catenin translocates into the nucleus, where it induces the transcription not only of cyclin D1 and c-myc, but also of a variety of other genes that contribute to cancer progression. These include the ECM protein fibronectin (33), which contributes to adhesion and motility; the metalloproteinase matrilysin (34), which stimulates extravasation and metastasis; and the multidrug resistance protein Mdr1 (35). In colorectal tumors, the central part of the tumor displays high levels of E-cad at the cell membrane that colocalizes with $\beta$-catenin. However, at the invasive front of these tumors, $\beta$-catenin is localized mainly in the nuclei of dissociated tumor cells that have lost E-cad expression (36). Interestingly, the loss of E-cad in such cells is apparently reversible and regulated by the tumor cell environment, since metastatic nodes from the same tumor display well-developed E-cad-containing AJs. Thus, the loss of E-cad that causes disruption of cell adhesion and polarity allows tumor cell metastasis, while the translocation of $\beta$-catenin into the nucleus might be required to induce the expression of genes that promote cell proliferation and invasion.

Activation of $\beta$-catenin signaling also occurs during EMT mediated either by c-Fos or by the IGF II signaling pathway. Tyrosine phosphorylation of $\beta$-catenin, which promotes disruption of AJs, also increases the affinity of $\beta$-catenin to the TATA-binding protein, a general positive regulator of transcription (37). Nevertheless, analysis of breast cancer cell lines lacking cadherin expression does not support a correlation between loss of E-cad expression and activation of $\beta$-catenin signaling (38). Additional events, including compromised proteasomal degradation of $\beta$-catenin, its tyrosine phosphorylation, and possibly a release from transcriptional inhibition, may be required to activate $\beta$-catenin signaling in such cancer cells.

\section{Cross-talk between AJs and growth factor receptors}

Growth factors such as EGF, HGF/scatter factor (SF), and FGF can induce the dismantling of AJs and can cause a dramatic change in cell morphology and gene expression in which cells shift from an epithelial to a fibroblastic phenotype and initiate a program of invasive growth $(39,40)$. Such growth factors operate via receptor tyrosine kinases, which often localize to sites of AJ assembly, as demonstrated for the EGF and HGF receptors. It is conceivable that these activated growth factor receptors tyrosine-phosphorylate various AJ components, including $\beta$-catenin, thereby contributing to the disruption of AJs observed in cancer cells. For example, the poor AJ organization of some Ras-transformed cells has been attributed to enhanced tyrosine phosphorylation of $\beta$-catenin (41), which, at least in vitro, results in its decreased binding to cadherin (42). Disruption of AJs by HGF stimulation requires, in addition, the activation of phosphatidylinositol (PI) 3-kinase and mitogen-activated protein kinase (MAPK) by HGF/SF, since inhibition of these kinases prevents the disassembly of AJs in these cells (43). The regula- 
tion of AJ assembly may also be achieved via phosphorylation by CKII on serine residues of the cadherin cytoplasmic tail, a modification that enhances its binding to $\beta$-catenin (44). Point mutations in the phosphorylation sites of the cadherin cytoplasmic tail have been shown to reduce its binding to $\beta$-catenin $(44,45)$.

Whereas tyrosine kinase receptor signaling regulates cell adhesion, it is also possible that AJs, in turn, influence tyrosine kinase signaling. The reduction in cell proliferation characteristic of dense cell cultures is mediated, in part, by cadherin-containing AJs that render the cells insensitive to growth factor stimulation. For example, in dense cultures that assemble extensive AJs, liganddependent dimerization and activation of the EGF receptor tyrosine kinase are inhibited (46). In addition, in cells maintained in a three-dimensional culture system, cadherin-dependent growth inhibition is mediated by the activation of tyrosine phosphatases, which suppress signaling pathways initiated by receptor tyrosine kinases. In such cells, the assembly of AJs prevents the activation of the EGF receptor pathway by TGF- $\alpha$ (47).

Interestingly, $\mathrm{N}$-cadherin, in contrast to E-cadherin, enhances tyrosine kinase signaling and thus enhances cell motility. High levels of $\mathrm{N}$-cadherin have been found in invasive tumor cell lines $(48,49)$. The ability of $\mathrm{N}$-cadherin to enhance cell motility, invasion, and metastasis is apparently due to the activation of FGF receptor signaling and involves its interaction with the extracellular domain of $\mathrm{N}$-cadherin $(48,49)$.

\section{Rho GTPases and the regulation of cell-cell adhesion}

Small GTPases of the Rho family (Rho, Rac, and $\mathrm{Cdc} 42$ ) are regulatory proteins that coordinate remodeling of the actin cytoskeleton in response to various stimuli (50). In the active, GTP-bound form, they interact with and activate target proteins that regulate actin polymerization, cell motility, and gene expression. The Rho GTPases are involved in cell transformation, and some of their activators are oncogenes, including Vav, $\mathrm{Net}$, and $\mathrm{Dbl}$ (51). The assembly and maintenance of AJs require the activity of Rho and Rac $(52,53)$, and deregulation of these small GTPases in transformed cells has been shown to interfere with cadherin function. For example, in Ras-transformed cells that are inefficient in the assembly of AJs, expression of Tiam1, a Rac activator, can restore AJ assembly and epithelial morphology and can inhibit invasiveness (54). While the molecular mechanisms by which small GTPases affect cell-cell adhesion are still poorly understood, they likely involve regulating actin cytoskeletal organization and $\mathrm{AJ}$ assembly (55).

\section{Conclusions and future perspectives}

The assembly of cadherin-containing AJs is essential for the formation and maintenance of cellular and tissue integrity. In addition to their structural-mechanical function, these junctions play a pivotal role in regulating cellular responses to adhesion- and growth factor-mediated cues, and other environmental signals. The ability of excessive AJ assembly to decrease cell growth and motility is probably related to the action of AJs as tumor suppressors. During cancer progression, cells evade adhesion-mediated regulatory mechanisms by either acquiring mutations in $\mathrm{AJ}$ proteins, or inactivating their function by various signaling mechanisms. The loss of AJs in cooperation with deregulation of other pathways leads to enhanced cell proliferation, motility, and, eventually, metastasis.

The mechanisms regulating the assembly of AJs and those involved in cadherin-mediated signaling via $\beta$-catenin are poorly understood. Future efforts will probably be devoted to understanding the details of how AJs are disrupted in response to MAPK or PI3kinase activation and to changes in the activity of the small GTPases of the Rho family. The signaling pathways that repress E-cad expression through the transcription factors snail, E12/E47, and others, as well as their regulation in cancer cells, require further study. These subjects are currently under intensive investigation, and we expect that such studies will provide new information that will allow the identification of novel targets for inhibiting tumor development.

\section{Acknowledgments}

The studies from the authors' laboratory are supported by a grant from the Israel Science Foundation.

1. Ben-Ze'ev, A., and Geiger, B. 1998. Differential molecular interactions of $\beta$-catenin and plakoglobin in adhesion, signaling and cancer. Curr. Opin. Cell Biol. 10:629-639.

2. Nagafuchi, A. 2001. Molecular architecture of adherens junctions. Curr Opin. Cell Biol. 13:600-603.

3. Perl, A., Wilgenbus, P., Dahl, U., Semb, H., and Christofori, G. 1998. A causal role for E-cadherin in the transition from adenoma to carcinoma. Nature. 392:190-193.

4. Vleminckx, K., Vakaet, L., Mareel, M., Fiers, W., and van Roy, F. 1991. Genetic manipulation of E-cadherin expression by epithelial tumor cells reveals an invasion suppressor role. Cell. 66:107-119.

5. Hirohashi, S. 1998. Inactivation of the E-cadherin-mediated cell adhesion system in human cancers. Am. J. Pathol. 153:333-339.

6. Jones, P., and Laird, P. 1999. Cancer-epigenetics comes of age. Nat. Genet. 21:163-167.

7. Graff, J.R., Gabrielson, E., Fujii, H., Baylin, S.B., and Herman, J.G. 2000 Methylation patterns of the E-cadherin $5^{\prime} \mathrm{CPG}$ island are unstable and reflect the dynamic, heterogeneous loss of E-cadherin expression during metastatic progression. J. Biol. Chem. 275:2727-2732.

8. Grady, W., et al. 2000. Methylation of the CDH1 promoter as the second genetic hit in hereditary diffuse gastric cancer. Nat. Genet. 26:16-17.

9. Cano, A., et al. 2000. The transcription factor snail controls epithelialmesenchymal transitions by repressing E-cadherin expression. Nat. Cell Biol. 2:76-83.

10. Batlle, E., et al. 2000. The transcription factor snail is a repressor of Ecadherin gene expression in epithelial tumour cells. Nat. Cell Biol. 2:84-89.

11. Comijn, J., et al. 2001. The two-handed E box binding zinc finger protein SIP1 downregulates E-cadherin and induces invasion. Mol. Cell. 7:1267-1278

12. Perez-Moreno, M., et al. 2001. A new role for E12/E47 in the repression of E-cadherin expression and epithelial-mesenchymal transitions. J. Biol. Chem. 276:27424-27431.

13. Palmer, H.G., et al. 2001. Vitamin D(3) promotes the differentiation of colon carcinoma cells by the induction of E-cadherin and the inhibition of $\beta$-catenin signaling. J. Cell Biol. 154:369-387.

14. Hirano, S., Kimoto, N., Shimoyama, Y., Hirohashi, S., and Takeichi, M 1992. Identification of a neural $\alpha$-catenin as a key regulator of cadherin function and multicellular organization. Cell. 70:293-301.

15. Bullions, L., Notterman, D., Chung, L., and Levine, A. 1997. Expression of wild-type $\alpha$-catenin protein in cells with a mutant $\alpha$-catenin gene restores both growth regulation and tumor suppressor activities. Mol. Cell. Biol. 17:4501-4508.

16. Vasioukhin, V., Bauer, C., Degenstein, L., Wise, B., and Fuchs, E. 2001. Hyperproliferation and defects in epithelial polarity upon conditional 
ablation of $\alpha$-catenin in skin. Cell. 104:605-617.

17. Bienz, M., and Clevers, H. 2000. Linking colorectal cancer to Wnt signaling. Cell. 103:311-320.

18. Polakis, P. 2000. Wnt signaling and cancer. Genes Dev. 14:1837-1851.

19. Heasman, J., et al. 1994. Overexpression of cadherins and underexpression of $\beta$-catenin inhibit dorsal mesoderm induction in early Xenopus embryos. Cell. 79:791-803.

20. Fagotto, F., Funayama, N., Gluck, U., and Gumbiner, B. 1996. Binding to cadherins antagonizes the signaling activity of $\beta$-catenin during axis formation in Xenopus. J. Cell Biol. 132:1105-1114.

21. Sadot, E., Simcha, I., Shtutman, M., Ben-Ze'ev, A., and Geiger, B. 1998. Inhibition of $\beta$-catenin-mediated transactivation by cadherin derivatives. Proc. Natl. Acad. Sci. USA. 95:15339-15344.

22. Simcha, I., et al. 1998. Differential nuclear translocation and transactivation potential of $\beta$-catenin and plakoglobin. J. Cell Biol. 141:1433-1448.

23. Shtutman, M., et al. 1999. The cyclin D1 gene is a target of the $\beta$-catenin/LEF-1 pathway. Proc. Natl. Acad. Sci. USA. 96:5522-5527.

24. Tetsu, O., and McCormick, F. 1999. $\beta$-catenin regulates expression of cyclin D1 in colon carcinoma cells. Nature. 398:422-426.

25. He, T., et al. 1998. Identification of c-MYC as a target of the APC pathway. Science. 281:1509-1512.

26. Gottardi, C., Wong, E., and Gumbiner, B. 2001. E-cadherin suppresses cellular transformation by inhibiting $\beta$-catenin signaling in an adhesion independent manner. J. Cell Biol. 153:1049-1060.

27. Stockinger, A., Eger, A., Wolf, J., Beug, H., and Foisner, R. 2001. E-cadherin regulates cell growth by modulating proliferation-dependent $\beta$-catenin transcriptional activity. J. Cell Biol. 154:1185-1196.

28. Damalas, A., et al. 1999. Excess $\beta$-catenin promotes accumulation of transcriptionally active p53. EMBO J. 18:3054-3063.

29. Damalas, A., Kahan, S., Shtutman, M., Ben-Ze'ev, A., and Oren, M. 2001 Deregulated $\beta$-catenin induces a p53-and ARF-dependent growth arrest and cooperates with Ras in transformation. EMBO J. 20:4912-4922.

30. Sadot, E., Geiger, B., Oren, M., and Ben-Ze'ev, A. 2001. Down-regulation of $\beta$-catenin by activated p53. Mol. Cell. Biol. 21:6768-6781.

31. Liu, J., et al. 2001. Siah-1 mediates a novel $\beta$-catenin degradation pathway linking p53 to the adenomatous polyposis coli protein. Mol. Cell. 7:927-936.

32. Matsuzawa, S.I., and Reed, J.C. 2001. Siah-1, SIP, and Ebi collaborate in a novel pathway for $\beta$-catenin degradation linked to 53 responses. Mol. Cell. 7:915-926.

33. Gradl, D., Kuhl, M., and Wedlich, D. 1999. The Wnt/Wg signal transducer $\beta$-catenin controls fibronectin expression. Mol. Cell. Biol. 19:5576-5587.

34. Crawford, H., et al. 1999. The metalloproteinase matrilysin is a target of $\beta$-catenin transactivation in intestinal tumors. Oncogene. 18:2883-2891.

35. Yamada, T., et al. 2000. Transactivation of the multidrug resistance 1 gene by $\mathrm{T}$-cell factor $4 / \beta$-catenin complex in early colorectal carcinogenesis. Cancer Res. 60:4761-4766

36. Brabletz, T., et al. 2001. Variable $\beta$-catenin expression in colorectal cancers indicates tumor progression driven by the tumor environment. Proc. Natl. Acad. Sci. USA. 98:10356-10361.
37. Piedra, J., et al. 2001. Regulation of $\beta$-catenin structure and activity by tyrosine phosphorylation. J. Biol. Chem. 276:20436-20443.

38. van de Wetering, M., et al. 2001. Mutant E-cadherin breast cancer cells do not display constitutive Wnt signaling. Cancer Res. 61:278-284.

39. Savagner, P. 2001. Leaving the neighborhood: molecular mechanisms involved during epithelial-mesenchymal transition. Bioessays. 23:912-923.

40. Hay, E. 1995. An overview of epithelio-mesenchymal transformation. Acta Anat. (Basel). 154:8-20.

41. Kinch, M., Clark, G., Der, C., and Burridge, K. 1995. Tyrosine phosphorylation regulates the adhesions of ras-transformed breast epithelia. J. Cell Biol. 130:461-471.

42. Roura, S., Miravet, S., Piedra, J., Garcia de Herreros, A., and Dunach, M. 1999. Regulation of E-cadherin/Catenin association by tyrosine phosphorylation. J. Biol. Chem. 274:36734-36740.

43. Potempa, S., and Ridley, A. 1998. Activation of both MAP kinase and phosphatidylinositide 3-kinase by Ras is required for hepatocyte growth factor/scatter factor-induced adherens junction disassembly. Mol. Biol. Cell. 9:2185-2200.

44. Lickert, H., Bauer, A., Kemler, R., and Stappert, J. 2000. Casein kinase II phosphorylation of E-cadherin increases E-cadherin/ $\beta$-catenin interaction and strengthens cell-cell adhesion. J. Biol. Chem. 275:5090-5095.

45. Simcha, I., et al. 2001. Cadherin sequences that inhibit $\beta$-catenin signaling: a study in yeast and mammalian cells. Mol. Biol. Cell. 12:1177-1188.

46. Takahashi, K., and Suzuki, K. 1996. Density-dependent inhibition of growth involves prevention of EGF receptor activation by E-cadherinmediated cell-cell adhesion. Exp. Cell Res. 226:214-222.

47. St. Croix, B., et al. 1998. E-Cadherin-dependent growth suppression is mediated by the cyclin dependent kinase inhibitor p27(KIP1).J. Cell Biol. 142:557-571.

48. Nieman, M., Prudoff, R., Johnson, K., and Wheelock, M. 1999. N-cadherin promotes motility in human breast cancer cells regardless of their E-cadherin expression. J. Cell Biol. 147:631-644.

49. Hazan, R., Phillips, G., Qiao, R., Norton, L., and Aaronson, S. 2000 Exogenous expression of $\mathrm{N}$-cadherin in breast cancer cells induces cell migration, invasion, and metastasis. J. Cell Biol. 148:779-790.

50. Ridley, A. 2001. Rho family proteins: coordinating cell responses. Trends Cell Biol. 11:471-477.

51. Van Aelst, L., and D'Souza-Schorey, C. 1997. Rho GTPases and signaling networks. Genes Dev. 11:2295-2322.

52. Braga, V., Machesky, L., Hall, A., and Hotchin, N. 1997. The small GTPases Rho and Rac are required for the establishment of cadherin-dependent cell-cell contacts. J. Cell Biol. 137:1421-1431.

53. Takaishi, K., Sasaki, T., Kotani, H., Nishioka, H., and Takai, Y. 1997. Regulation of cell-cell adhesion by rac and tho small G proteins in MDCK cells. J. Cell Biol. 139:1047-1059.

54. Hordijk, P., et al. 1997. Inhibition of invasion of epithelial cells by Tiam 1-Rac signaling. Science. 278:1464-1466.

55. Fukata, M., and Kaibuchi, K. 2001. Rho-family GTPases in cadherinmediated cell-cell adhesion. Nat. Rev. Mol. Cell Biol. 2:887-897.

56. Zhurinsky, J., Shtutman, M., and Ben-Ze'ev, A. 2000. Plakoglobin and $\beta$-catenin: protein interactions, regulation and biological roles. J. Cell Sci. 113:3127-3139. 